\title{
TTF-PTM dyads: From switched molecular self assembly in solution to radical conductors in solid state
}

Manuel Souto, Concepció Rovira, Imma Ratera, and Jaume Veciana

Organic donor-acceptor (D-A) systems formed by the electron-donor tetrathiafulvalene (TTF) linked to the electron-acceptor polychlorotriphenylmethyl (PTM) radical through different $\pi$-conjugated bridges exhibit interesting physical properties such as bistability in solution or conductivity in solid state. Understanding the interplay between intra- and intermolecular charge transfer processes in solution is of high interest in order to rationalize the selfassembling ability and conducting properties of such dyads in solid state. In this Highlight we go over the self-assembling properties of different TTF- $\pi$-PTM radical dyads that find potential applications as molecular switches or conductors in the field of molecular electronics.

\section{Introduction}

During the last decades the molecular approach to material science has emerged as a promising research field for the generation of new electronic devices at the nanoscale level because of their chemical and physical peculiarities. ${ }^{1}$ Owing to important perspectives in both fundamental sciences and applications in molecular electronics, numerous efforts have been made to design new multifunctional materials which involve the interplay between multiple physical properties (i.e. electrical, optical, and magnetic properties). ${ }^{2}$

Multifunctional molecular materials can be even more interesting if they are able to switch between two or more states modifying their physical properties (i.e. optical, magnetic or electrical properties) upon the application of external stimuli (i.e. temperature, ${ }^{3}$ pressure, ${ }^{4}$ light ${ }^{5}$ or electric field $\left.{ }^{6}\right)$. This interest of such phenomenon is mainly due to their potential application as molecular switches and memories. ${ }^{7,8}$ Some of molecular materials exhibiting bistability are, for example, spin-crossover, ${ }^{9}$ valence-tautomeric ${ }^{10}$ or donor-acceptor (D-A) systems. ${ }^{11}$

In this direction, there are some stable radicals that can exhibit a reversible dimerization in response to external stimuli, with monomers and dimers coexisting at the equilibrium in solution ${ }^{12}$ and may produce bistable systems in the solid state. ${ }^{13-15}$ Spirobisphenalenyl $\left.\right|^{16,17}$ and thiazyl ${ }^{18,19}$ radicals are good examples of such materials (Fig. 1). These radicals are able to switch between a paramagnetic monomeric radical form at high temperature and a diamagnetic dimeric form at lower temperature that mainly arises from the $\pi-\pi$ intermolecular interactions within the dimers. These kind of radical molecules are interesting not only because the supramolecular bistability phenomenon in solution but also due to their potential use as single-component conductors once crystallized. Indeed, it was proposed that neutral organic radicals could be used as molecular conductors since the unpaired electron can serve as charge carrier. ${ }^{20}$ However, most of organic radicals exhibit large Coulomb energy repulsion $U$ and narrow electronic bandwidth $W$ giving rise to a Mott insulator behavior. For this reason, most of the reported examples of neutral radical conductors are based on highly delocalized systems in order to decrease the repulsion energy $U$ or incorporate heteroatoms to enhance the electronic bandwidth $W .^{21-26}$

a)

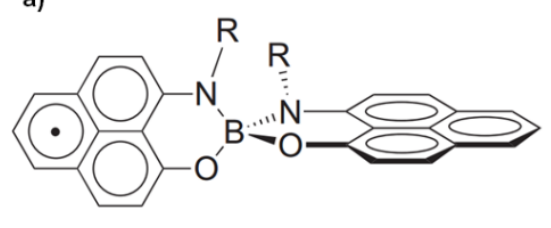

b)<smiles></smiles>

Fig. 1. Molecular structures of a) spirobisphenalenyl radical $(\mathrm{R}=n-\mathrm{Bu})$ and b) bis $\left(1,2,3\right.$-dithiazolyl) radical $\left(\mathrm{R}^{1}=\mathrm{Et}, \mathrm{R}^{2}=\mathrm{F}\right)$.

Organic Donor-Acceptor (D-A) systems. Organic molecules formed by an electron donor (D) and an electron acceptor (A) units covalently linked by a $\pi$-conjugated bridge (D- $\pi-A)$ (Fig. 2) are an interesting family of molecules widely investigated for several applications, ranging from organic light-emitting devices, ${ }^{27}$ nonlinear optics, ${ }^{28}$ molecular electronics ${ }^{29}$ and photonics. ${ }^{30}$ D-A dyads are also very interesting materials due to the possibility of switching between different electronic states through variation of an external stimulus such as temperature, light or pressure. Indeed, when the appropriate external stimulus is applied, an intramolecular electron transfer (IET) process can be induced with the movement of an electron from the donor to the acceptor forming the charge-separated (zwitterionic) state that will show different physical properties (i.e. electrical, optical or magnetic properties) (Fig. 1). Thus, donor-acceptor systems could be 
potentially used as molecular switches due to the related bistability phenomenon. ${ }^{31}$ Otherwise, D-A dyads can also be of high interest if they exhibit a molecular packing in the solid state with complete segregation of donor and acceptor units exhibiting a good overlap between the molecules as it is a prerequisite for obtaining organic conductors, semiconductors or organic field-effect transistors (OFETs) that could exhibit ambipolar characteristics. ${ }^{32-34}$

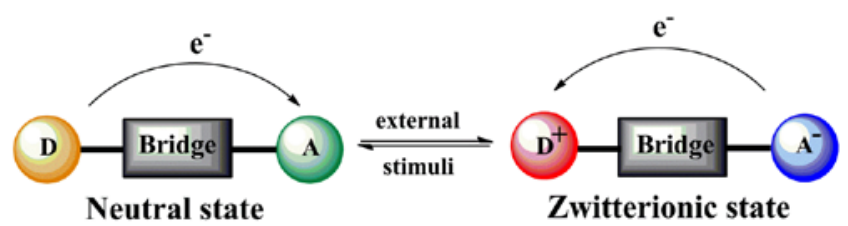

Fig. 2. Schematic drawing of a D-A dyad and associated bistability phenomenon when an external stimulus (i.e. $T, P$ or light) is applied forming the zwitterionic state with different physical properties.

\section{Building blocks: TTF and PTM radical.}

The electron-donor tetrathiafulvalene (TTF) and the electron-acceptor polychlorotriphenylmethyl (PTM) radical molecules are very good candidates as molecular building blocks for obtaining multifunctional D-A systems, since they allow the combination of magnetic, optical, conducting and electrochemical properties. Indeed, both units present different structural and electronic characteristics that make them interesting to build new multifunctional molecular materials.

TTF unit. Tetrathiafulvalene and its various derivatives are among the most versatile molecules which exhibit interesting redox properties and remarkable electron donor character. ${ }^{35,36}$ TTF is a non-aromatic $14-\pi$-electron system in which oxidation to the radical cation and dication species occurs sequentially and reversibly at very low oxidation potentials $\left(\mathrm{E}_{1 / 2}{ }^{1}=0.37 \mathrm{~V}\right.$ and $\mathrm{E}_{1 / 2}{ }^{2}=0.67 \mathrm{~V}$ in $\left.\mathrm{CH}_{2} \mathrm{Cl}_{2}\right)(\mathrm{Fig}$. 3$)$. These organosulfur molecules became of high interest due to their potential application in molecular electronics as semiconductors, superconductors and organic metals ${ }^{37}$ after the first synthesis of TTF reported by Wudl in $1970 .^{38}$ Since then several TTF derivatives have been reported finding applications in molecular and supramolecular structures, in switchable processes, ${ }^{39}$ as electron-donor unit in D-A systems ${ }^{33,40}$ or for the preparation of unimolecular rectifiers on surfaces. ${ }^{41}$ Also it has been reported several studies on the dimerisation phenomenon in solution have been reported in which the TTF is in the oxidized radical cation state. ${ }^{42-44}$

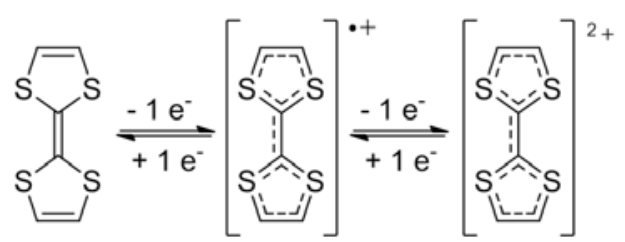

Fig. 3. TTF and its radical cation and dication derivative species.

PTM radical unit. Polychlorotriphenylmethyl (PTM) radicals are neutral open-shell organic molecules with a net magnetic moment which are characterized by an extremely high persistence and stability. They are composed of three chlorinated phenyl rings connected to a central carbon with a $s p^{2}$ hybridization exhibiting a large chemical and thermal stability. The large persistence of these radicals is mainly due to the presence of bulky chlorine atoms located in ortho position providing a source of steric protection for the central carbon where the spin density is mainly localized. ${ }^{31,45}$ In addition, PTM radicals are also interesting because they are electroactive species that can be oxidized to the polychlorotriphenylcarbonium ion or reduced to the polychlorotriphenylmethyl-carbanion (Fig. 4). The reduction of PTM radicals usually occurs at very low potentials $\left(-0.2 \mathrm{~V}\right.$ in $\left.\mathrm{CH}_{2} \mathrm{Cl}_{2}\right)$ and it is more feasible than the corresponding oxidation that requires high potentials $\left(1.6 \mathrm{~V}\right.$ in $\mathrm{CH}_{2} \mathrm{Cl}_{2}$ ). Such evidence makes this type of radicals optimal as an electron-acceptor unit in D-A systems with strong nonlinear optical (NLO) responses or to study intramolecular electron transfer for making switchable multifunctional molecular materials since the magnetic, optical and electronic properties of these open-shell molecules can be switched on and off with an external electrochemical stimuli. ${ }^{31}$ 

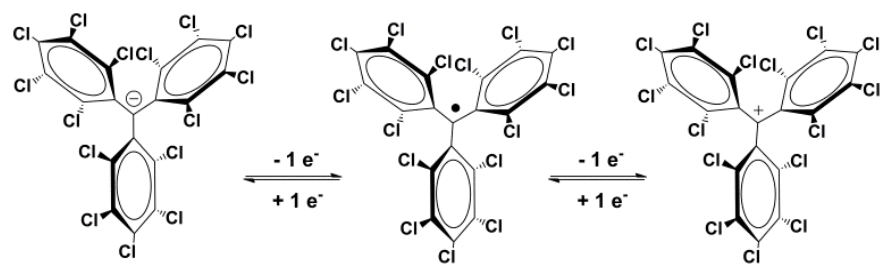

Fig. 4. PTM radical and its anion and cation derivative species.

In this Highlight we will review the recent developments in the synthesis and characterization of different TTF- $\pi$-PTM dyads which exhibit different physical properties such as optical, magnetic or electric depending on its molecular and supramolecular structure (Fig. 5). In particular, we will focus on the study of the interplay between the intra- and intermolecular charge transfer (CT) processes that plays a key role on the self-assembly of such dyads in solution in order to extend their physical properties and design molecular conductors in solid state.

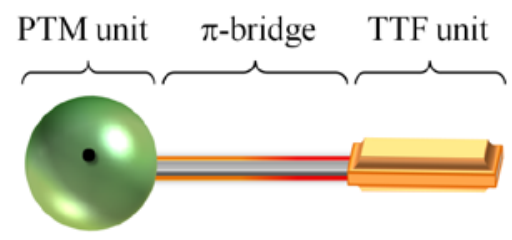

Fig. 5. Schematic representation of a TTF- $\pi$-PTM dyad.

\section{Studies in solution}

D-A radical dyads. Organic molecules that contain electron donor and electron acceptor units linked by $\pi$-conjugated bridges are very promising molecular materials for the development of molecular switches and memory devices. ${ }^{11}$ For example, during the last years in the framework of our group, it has been reported different D-A systems based on the electron-acceptor polychlorotriphenylmethyl (PTM) radical linked to ferrocene $(\mathrm{Fc})$ derivatives that can be switched from the neutral $(\mathrm{N})$ to the zwitterionic $(\mathrm{Z})$ state by changing the polarity of the solvent or by tuning the temperature in the solid state (Fig. 6). ${ }^{46-51}$ The cooperativity from electrostatic intermolecular interactions explains bistability observed in such D-A molecular crystals. ${ }^{48,52}$

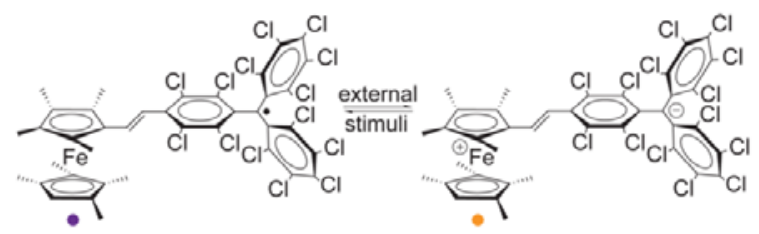

Fig. 6. Molecular structures relevant to the neutral D-A (purple, $\bullet$ ) and zwitterionic $D^{+}-A^{-}$(orange, $\bullet$ ) states of Me ${ }_{8}$ Fc-PTM derivative. Adapted with permission from ref. 50. Copyright (2013) American Chemical Society.

On the other hand, Nishida et al. proposed a TTF-based dyad which exhibits bistability in solution originated by an IET process between a TTF electron-donor linked to the electron-acceptor di-tert-butyl-6-oxophenalenoxyl (6OP) organic radical (Fig. 7). ${ }^{53}$ Such bistability was induced by changes in temperature and in the polarity of the solvent. Indeed, the phenomenon was followed by UV-vis-NIR spectroscopy observing the neutral state in $\mathrm{CH}_{2} \mathrm{Cl}_{2}$ whereas the zwitterionic state of the molecule was obtained in the polar $\mathrm{CF}_{3} \mathrm{CH}_{2} \mathrm{OH}$ solvent. 


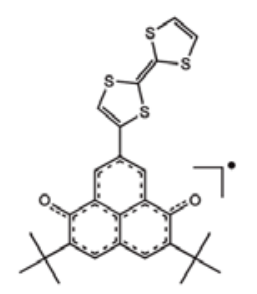

Neutral DA

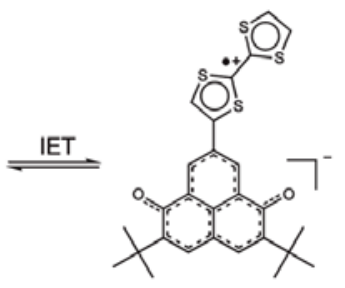

Zwitterionic $\mathrm{D}^{+\mathrm{A}^{-}}$

Fig. 7. The two possible states (neutral and zwitterionic) of the TTF-6OP dyad. Adapted with permission from ref. 53; Copyright 2005, John Wiley and sons.

Self-assembly of a TTF-PTM radical dyad (1). In a similar approach to the previous examples, in our group we have reported a D-A dyad that consists on the electron-acceptor PTM radical linked to a TTF through a vinylene bridge (1)..$^{54}$ This organic D-A radical dyad exhibited a reversible switching from its neutral to its zwitterionic state that can occur through an IET process from the TTF to the PTM radical simply by the modification of the solvent polarity (Fig. 8). For example, in the non-polar $\mathrm{CH}_{2} \mathrm{Cl}_{2}$ solvent only the neutral (1a) species was present whereas in the polar DMF solvent an IET process was promoted and the zwitterionic (1) species was formed.
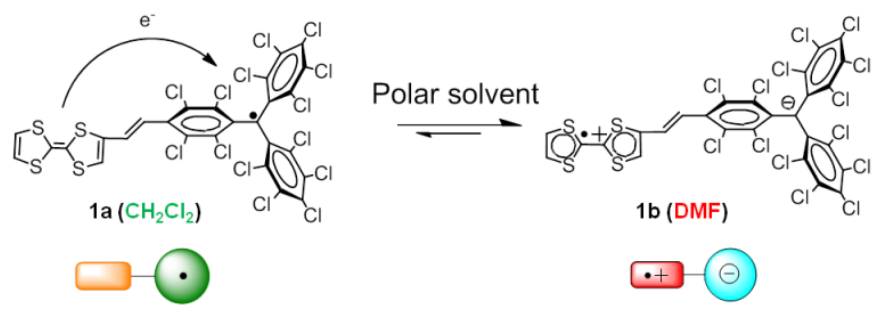

Fig. 8. Schematic representation of the reversible switching of the radical dyad TTF-PTM $\mathbf{1}$ between the neutral (1a) and zwitterionic (1b) states through an IET process.

This phenomenon was followed by UV-vis-NIR spectroscopy where the absorption spectrum of $\mathbf{1}$ in $\mathrm{CH}_{2} \mathrm{Cl}_{2}$ showed an intense band at $387 \mathrm{~nm}$ attributed to the radical chromophore of the PTM subunit whereas the spectrum in DMF does not show such a band but presented an intense band at $512 \mathrm{~nm}$ that corresponded to the anionic form of the PTM moiety (Fig. 9). Interestingly, the spectrum of 1 in acetone simultaneously exhibited the band located at $387 \mathrm{~nm}$ as well as a band at $505 \mathrm{~nm}$ indicating the coexistence of both species $\mathbf{1 a}$ and $\mathbf{1 b}$ in equilibrium in this solvent.

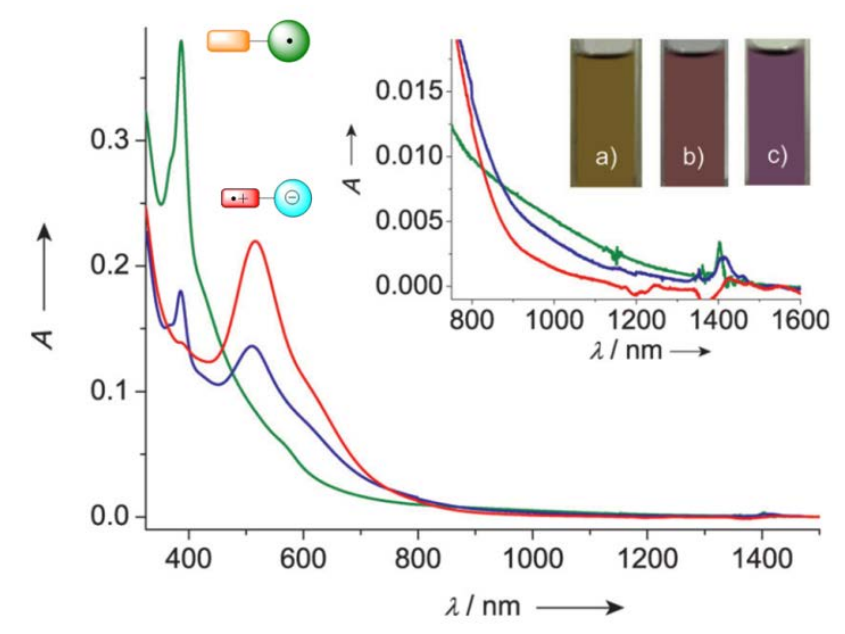


Fig. 9. UV-Vis-NIR spectra of dyad 1 in $\mathrm{CH}_{2} \mathrm{Cl}_{2}$ (green line), acetone (blue line), and DMF (red line). Inset: Low-energy range of the absorption spectra and colors of 1 exhibited in a) $\mathrm{CH}_{2} \mathrm{Cl}_{2}$, b) acetone, and c) DMF solutions. Adapted with permission from ref. 54; Copyright 2012, John Wiley and sons.

In addition, it was demonstrated that the IET process promoted the self-assembly of the dyads in the zwitterionic forms to form diamagnetic dimers in DMF at room temperature. Indeed, the ESR spectrum of dyad 1 in $\mathrm{CH}_{2} \mathrm{Cl}_{2}$ presented the typical signal for the PTM radical in agreement with the presence of neutral species whereas the spectrum in DMF does not give any signal (Fig. 10). The lack of signal related to the $\mathrm{TTF}^{+\cdot}$ cation radical suggested that zwitterionic species are aggregated forming diamagnetic dimers ( $\pi$-dimer) that are ESR silent at room temperature.

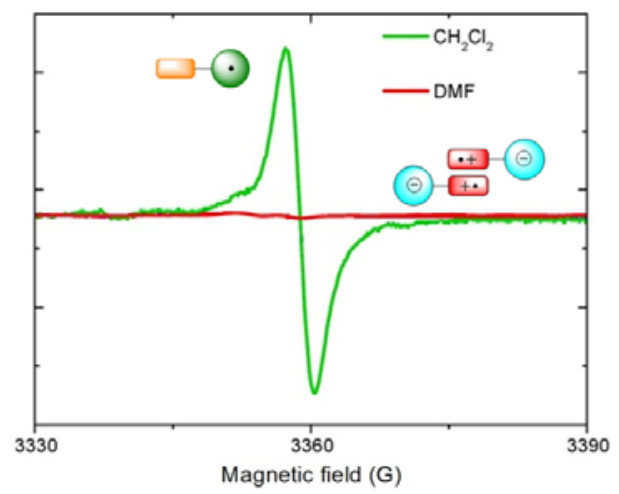

Fig. 10. ESR spectra of dyad 1 at $300 \mathrm{~K}$ in $\mathrm{CH}_{2} \mathrm{Cl}_{2}$ (green line) and DMF (red line). Schematic representation of monomers 1 a in $\mathrm{CH}_{2} \mathrm{Cl}_{2}$ and diamagnetic $\pi$-dimers of $\mathbf{1 b}$ in DMF solutions.

Thus, dyad 1 exhibits an intramolecular electron transfer phenomenon that enables the reversible switching from its neutral state 1a to its zwitterionic state $\mathbf{1 b}$ simply by modification of the solvent. In addition, the TTF-based self-assembly of this dyad was induced by the IET process between the donor and acceptor groups forming diamagnetic dimers of $\mathbf{1 b}$ in DMF solution.

Moreover, in order to investigate the origin of such aggregation phenomenon, temperature-dependent optical an ESR spectroscopies studies in $\mathrm{CH}_{2} \mathrm{Cl}_{2}$ were performed for a family of TTF-PTM dyads bearing different number of electrons (i.e. oxidizing the TTF subunit to $\mathrm{TTF}^{+\cdot}$ or reducing the $\mathrm{PTM}^{-}$radical subunit to $\mathrm{PTM}^{-}$anion) and/or with hydrogenated PTM residue (Fig. 11) giving information on the formation of homo- and mixed-valence dimers. ${ }^{55}$ Analysis of the equilibrium constants and thermodynamic parameters showed that dimers formed by radical dyads are much more stable due to the contribution of the intramolecular electron transfer that delocalizes the electrons along the vinylene bridge and the PTM subunit.

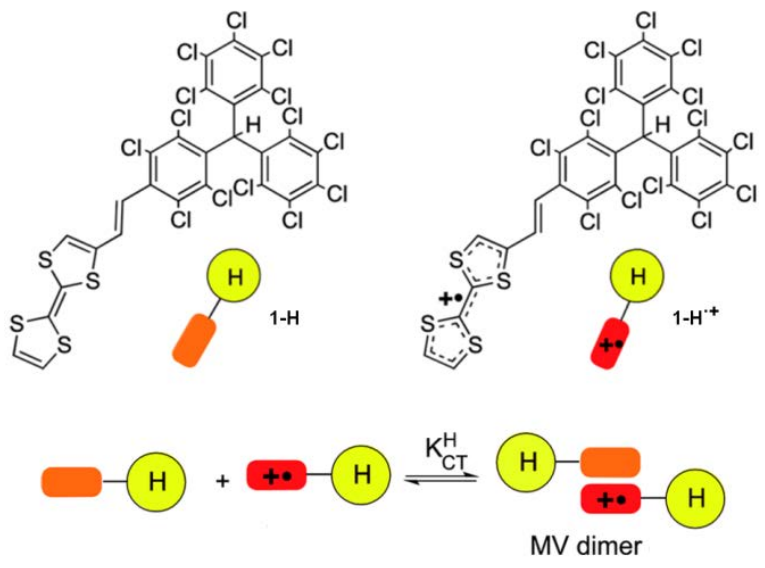

Fig. 11. Molecular structures of TTF-PTM dyads with hydrogenated PTM residue and with the partial oxidation of the TTF subunit. Schematic representation of the formation of the Mixed-Valence (MV) dimer.

Moreover, a theoretical model, merging a Hubbard-like description of the intermolecular charge transfer (ICT) interaction with a description of the intramolecular electron transfer (IET) in terms of a minimal model that just accounts for two essential electronic states 
(neutral and zwitterionic), was proposed in order to rationalize the subtle interplay between the IET within a dyad and the ICT occurring in a dimer between the TTF residues (Fig. 12).

\section{intermolecular CT \& IET}

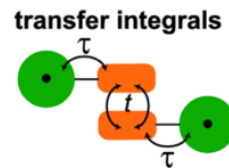

electron-electron repulsion energy
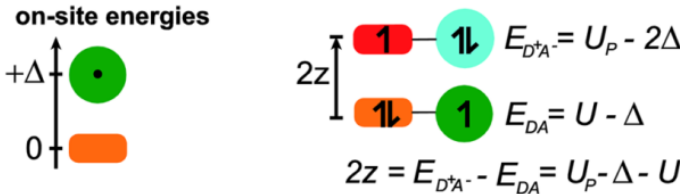

Fig. 12. Schematic picture of the model for intermolecular CT and intramolecular ET. Adapted with permission from ref. 55. Copyright (2013) American Chemical Society.

Thermomagnetic switch based on a TTF-PTM radical dyad (1). In order to get more insights on the dimerization of dyad 1 in DMF, we studied the reversible temperature-induced switching that exhibits radical dyad 1 in DMF solution between diamagnetic dimers [1 $]_{2}$ LT, observed at room temperature, and paramagnetic monomers $\mathbf{1}_{\mathrm{HT}}$ at high temperature (Fig. 13). ${ }^{56}$ This temperature-induced process was followed by different spectroscopic techniques such as UV-vis-NIR, ESR, Raman, ${ }^{1} \mathrm{H}-\mathrm{NMR}$ and cyclic voltammetry.

Regarding the optical properties, UV-vis-NIR spectra of dyad 1 in DMF were recorded at different temperatures in the $300-375 \mathrm{~K}$ range (Fig. 14) observing a clear color change. At $300 \mathrm{~K}$ the spectrum exhibited an intense band at $512 \mathrm{~nm}$ assigned to the PTM subunit in its anionic form indicating that $\mathbf{1}$ is only present in the zwitterionic $\mathrm{D}^{+} \mathrm{A}^{-}$state. When the solution was heated the band associated to the PTM anion weakens whereas the band related to the PTM neutral radical $(387 \mathrm{~nm})$ acquires intensity together with the appearance of a weak band at $950 \mathrm{~nm}$ that is associated to the IET process of $\mathbf{1}$ in the neutral D-A form.

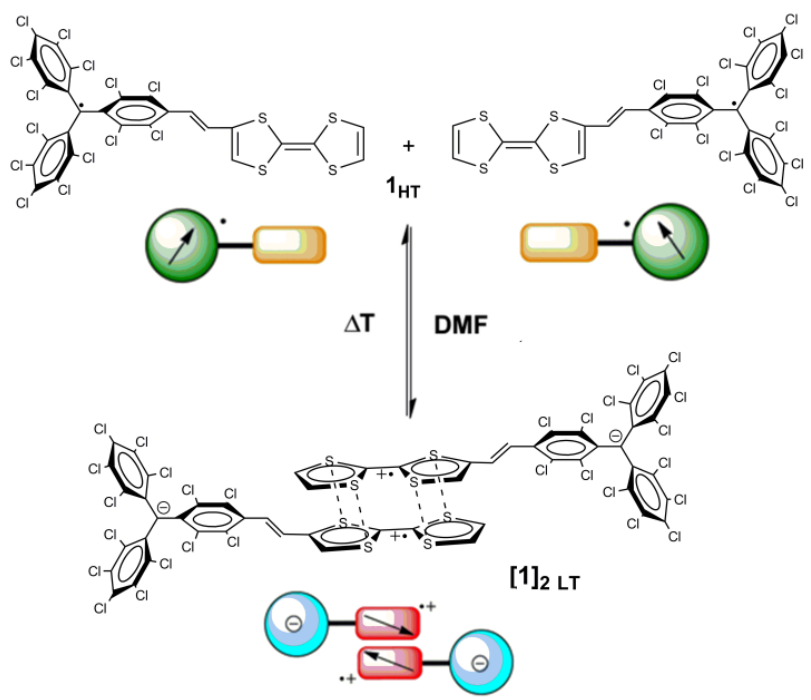

Fig. 13. Schematic representation of the reversible temperature-induced supramolecular switching of TTF-PTM (1) between diamagnetic dimers at low temperature $\left(\left[\mathbf{1}_{\mathbf{2}}\right.\right.$ LT $)$ and paramagnetic monomers at high temperature $\left(\mathbf{1}_{\mathrm{HT}}\right)$. Adapted with permission from ref. 56 . Copyright (2013) American Chemical Society. 


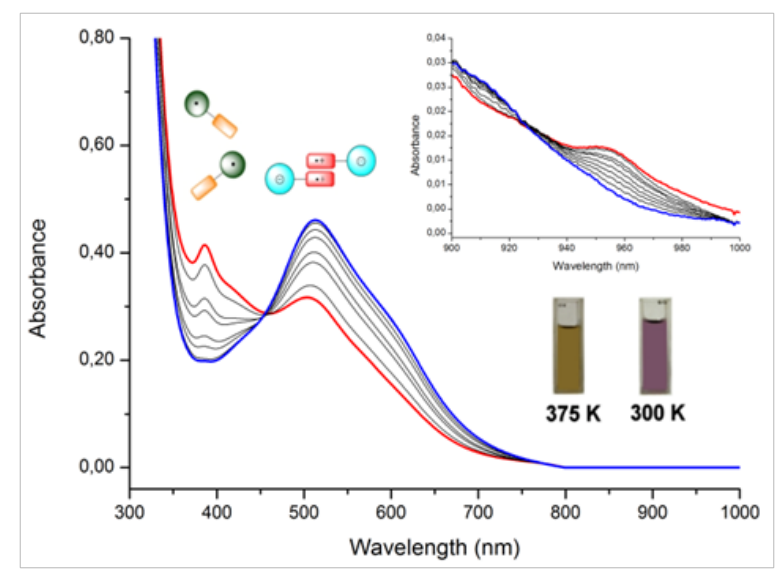

Fig. 14. UV-Vis spectra of dyad 1 in DMF (0.1 mM) at 375 (red line), 365, 355, 350, 345, 340, 330, 320 and $300 \mathrm{~K}$ (blue line). Adapted with permission from ref. 56. Copyright (2013) American Chemical Society.

On the other hand, ESR measurements were performed at different temperatures (Fig. 15). Whereas the strong antiferromagnetic coupling of TTF-radical spins in the $[\mathbf{1}]_{2 \mathrm{LT}}$ dimers is responsible for the lack of ESR signal at room temperature, heating the solution at 375 $\mathrm{K}$, a broad line appears at a $g$-value of 2.0025. This signal is typical of PTM radicals, indicating the formation of paramagnetic species that we identify as the $\mathbf{1}_{\mathrm{HT}}$ monomer in the neutral D-A form. Cooling down the solution to room temperature, it gradually becomes ESR silent again, exhibiting a completely reversible behavior from $375 \mathrm{~K}$ to $300 \mathrm{~K}$. Moreover, to demonstrate the complete reversibility of the system, several and consecutive ESR and UV-vis cycles at 300 and $375 \mathrm{~K}$ were performed without showing any sign of deterioration highlighting the reversibility to switch molecular self-assembly in this system.

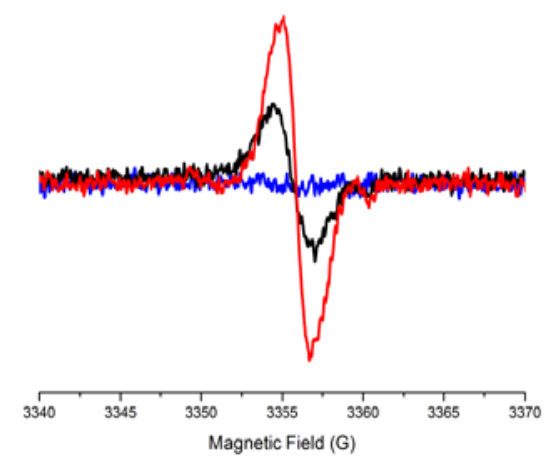

Fig. 15. ESR spectra of 1 in DMF (0.1 mM) at 375 (red line), 365 (black line) and 300 (blue line) K. Adapted with permission from ref. 56. Copyright (2013) American Chemical Society.

In view of the interesting electronic properties of some organic acceptor-donor-acceptor (A-D-A) triads, ${ }^{57-59}$ we have also studied the switching properties of a A-D-A diradical triad based on two PTM radicals connected through a TTF-vinylene bridge (2)..$^{60}$ This molecule exhibited an electrochemical reversible switching by one-electron reduction and oxidation modifying its optical, magnetic and electronic properties (Fig. 16). Indeed, we studied the generation of the mixed-valence radical anion $\mathbf{2}^{-}$and triradical cation $\mathbf{2}^{\cdots+}$ species obtained upon electrochemical reduction or oxidation, respectively, monitoring the properties by optical and ESR spectroscopy. Interestingly, the modification of electron delocalization and magnetic coupling was observed when the charged species were generated and these changes were rationalized by theoretical calculations. However, the dimerization process between the TTF moieties taking place in the TTF-PTM dyad was not observed in this case probably due to the steric hindrance produced by the additional PTM radical moiety. 


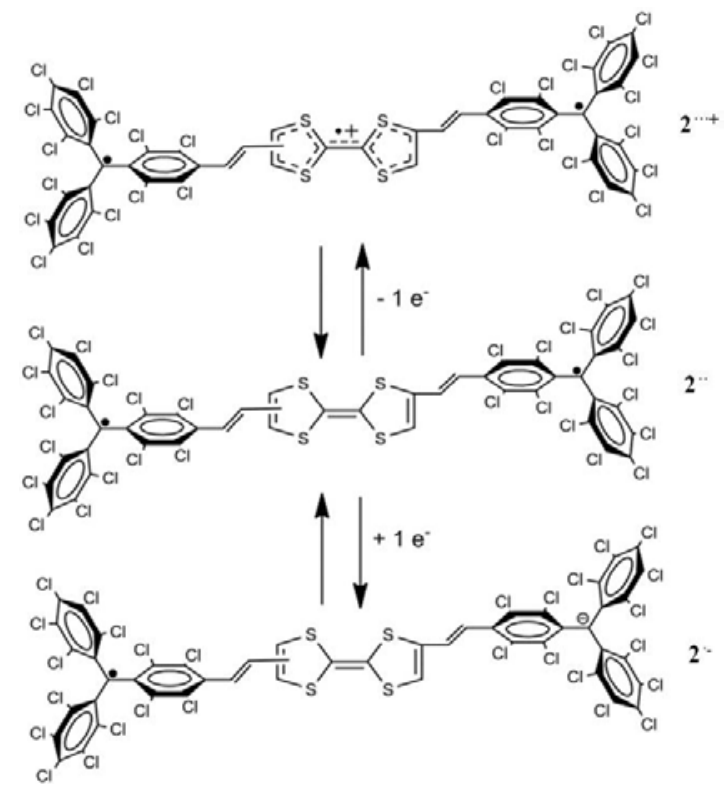

Fig. 16. Molecular structures of diradical 2, mixed-valence $\mathbf{2}^{--}$and triradical cation $\mathbf{2}^{\cdots++}$. Adapted with permission from ref. 60 . Copyright (2016) American Chemical Society.

\section{Studies in solid state}

After the understanding of such interesting physical properties and self-assembling abilities of TTF-PTM dyads in solution, we aimed to extend and exploit these properties in solid state. In fact, one of the main interests of these systems is to combine both the potential conducting properties of the partially oxidized TTF moiety and the magnetic properties of the PTM unit in a cooperative manner in order to develop new spin-polarized donor systems exhibiting magnetoresistance (change of the electrical resistance when applying a magnetic field). ${ }^{61,62}$ Moreover, the TTF unit can be considered as an ideal building block for improving the intermolecular interactions in order to exploit the conducting properties in the solid state. For this reason, first of all it is necessary to study in detail the crystal structures of different TTF-PTM dyads and analyze the different supramolecular arrangements.

Crystal structure of 1-H. The crystal structure of the hydrogenated derivative 1-H (Fig. 17) showed that it crystallizes in the triclinic system with a $\boldsymbol{P} \mathbf{1}$ space group. The molecular structure revealed that the vinylene bridge is in the trans-position and the crystal packing showed that molecules are arranged in a head-to-tail manner with short $\mathrm{S} \cdots \mathrm{Cl}(3.38 \AA$ ) and $\mathrm{Cl} \cdots \mathrm{Cl}(3.35 \AA$ A $)$ contacts (Fig. 18). Thus, this supramolecular arrangement was not optimal for developing conducting molecular materials. Unfortunately, up to date, it has not been possible to study in detail the properties in the solid state of radical dyad $\mathbf{1}$ because of the poor crystalline quality that prevented the determination of its $\mathrm{X}$-ray crystal structure.

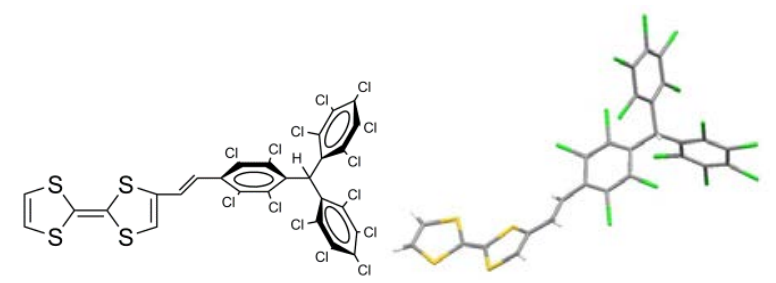

Fig. 17. Molecular structure and ORTEP views of 1-H. Atoms are shown at the $50 \%$ probability level. 


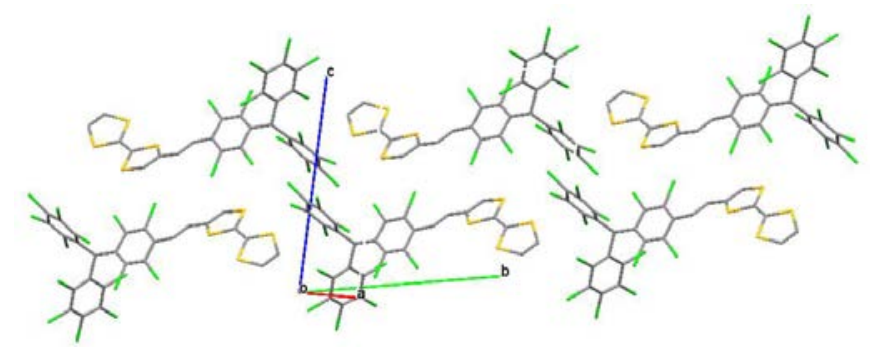

Fig. 18. Crystal packing of dyad $\mathbf{1 - H}$ in the $b c$ plane.

Crystal structure of MPTTF-PTM dyad (3 and 3-H). In order to improve the self-assembly of the TTF moiety of TTF-PTM dyads and improve their crystalline quality, we have designed and obtained the TTF-based radical dyad $\mathbf{3}$ with a longer bridge and higher degree of coplanarity between the TTF and PTM units than dyad 1. The increased length of the bridge was obtained using a monopyrrolo-TTF (MPTTF) building block connected through a conjugated phenyl-vinylene to the PTM unit obtaining the MPTTF-PTM dyad $\mathbf{3}$ and its hydrogenated derivative 3-H (Fig. 19). ${ }^{63}$
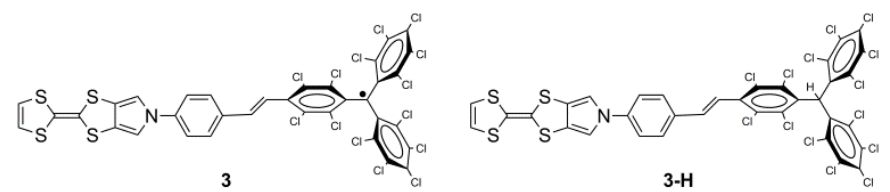

Fig. 19. Molecular structures of radical 3 and non-radical 3-H dyads.

X-ray diffraction analysis at $300 \mathrm{~K}$ on red crystals of 3-H indicates that it crystallizes in the triclinic system with a $\boldsymbol{P} \overline{\mathbf{1}}$ space group. The TTF moieties of neighboring molecules are forming alternated face-to-face dimers on the $b c$ plane forming 1-D chains along the $c$-axis with the shortest S-S distances of $3.92 \AA$ (Fig. 20). On the other hand, PTM units are also stacked forming monodimensional chains along the caxis with short intermolecular $\mathrm{Cl} \cdots \mathrm{Cl}$ interactions that are in the range of 3.28-3.47 $\AA$. This supramolecular arrangement was not optimal for obtaining conducting materials since the TTF units are not forming regular stacks and they are showing face-to-face dimers with large space between them.

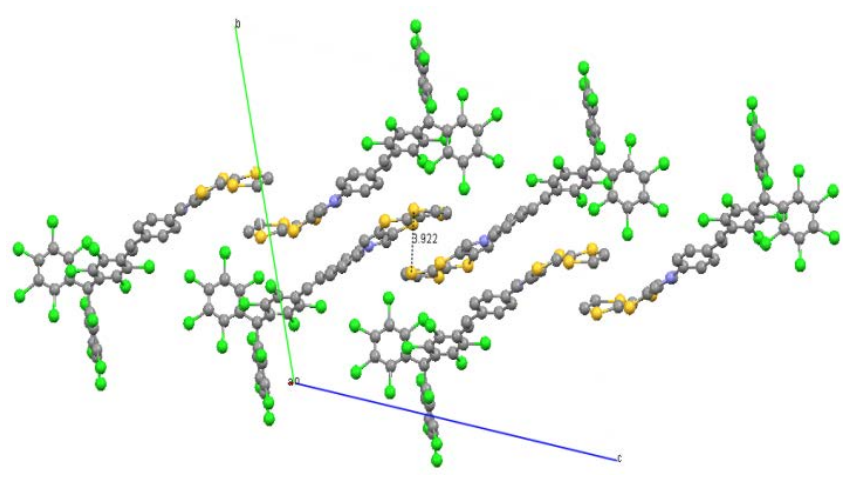

Fig. 20. Molecular packing of dyad 3-H in the bc plane. Adapted with permission from ref. 63; Copyright 2015, John Wiley and sons.

On the other hand, radical dyad $\mathbf{3}$ crystallizes in the monoclinic system with the space group $P 2_{1}$ and the asymmetric unit reveals two inequivalent molecules that are very similar in geometry. Regarding the molecular arrangement, molecules of radical dyad $\mathbf{3}$ are stacked forming 1D chains on the $a b$ plane in which MPTTF units are forming a herringbone structure with $\mathrm{S} \cdots \mathrm{S}$ and $\mathrm{Cl} \cdots \mathrm{Cl}$ interactions of 3.91 and $3.33 \AA$, respectively (Fig. 21). In view of such crystal structure with segregated donor and acceptor units we proposed that this system could be an optimal candidate for developing a single-component conductor if improving the intermolecular interactions and doping the TTF moieties. 


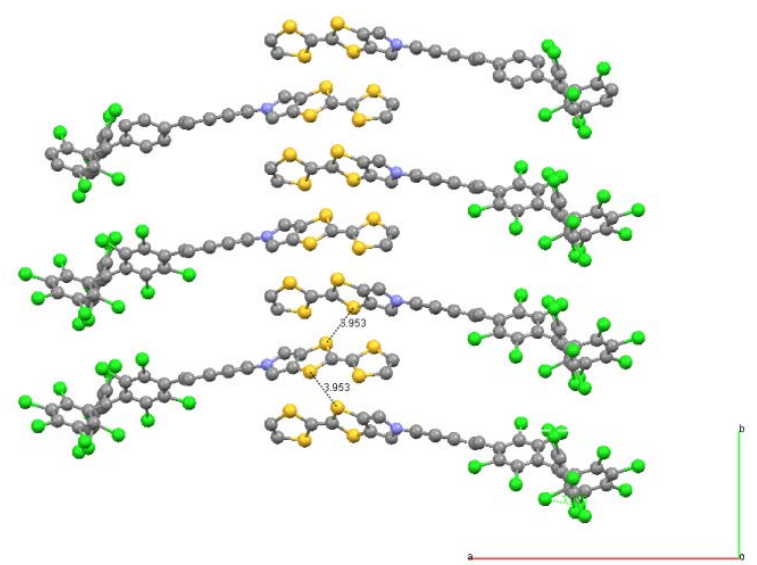

Fig. 21. Molecular packing of radical dyad 3 in the $b c$ plane. Adapted with permission from ref. 63; Copyright 2015, John Wiley and sons.

Molecular radical conductor in solid state. In view of the supramolecular architecture of radical dyad $\mathbf{3}$ with segregated donor and acceptor units we aimed to promote conductivity upon the application of high pressure since we could enhance the electronic bandwidth $W$ of the system. Indeed, we have recently reported the resistivity measurements of dyad $\mathbf{3}$ at high pressure indicating semiconductor behaviour and a detailed study on the origin of the conductivity at high pressure performed by theoretical calculations as well as Raman spectroscopy at different pressures. ${ }^{64}$

Resistivity measurements of crystals of radical dyad $\mathbf{3}$ were performed up to $21 \mathrm{GPa}$ along the $b$-axis. Crystals of $\mathbf{3}$ showed insulating behaviour at ambient pressure whereas they exhibited semiconducting behaviour increasing the pressure from $6.5 \mathrm{GPa}$ (Fig. 22). The roomtemperature conductivity of 3 at $15 \mathrm{GPa}$ was found to be $0.76 \mathrm{~S} \mathrm{~cm}^{-1}$ with a low activation energy $\left(E_{\mathrm{a}}\right)$ of $0.067 \mathrm{eV}$.

On the other hand, crystals of the PTM radical without any substituent exhibited insulating behaviour at all measured pressures. This different behaviour was related to the shorter distances between the molecules in the crystal structure of $\mathbf{3}$ in comparison with unsubstituted PTM due to the short S...S intermolecular interactions between the TTF moieties. Thus, the electronic bandwidth $W$ was increased in compound $\mathbf{3}$ in comparison with the PTM radical without any substituent.

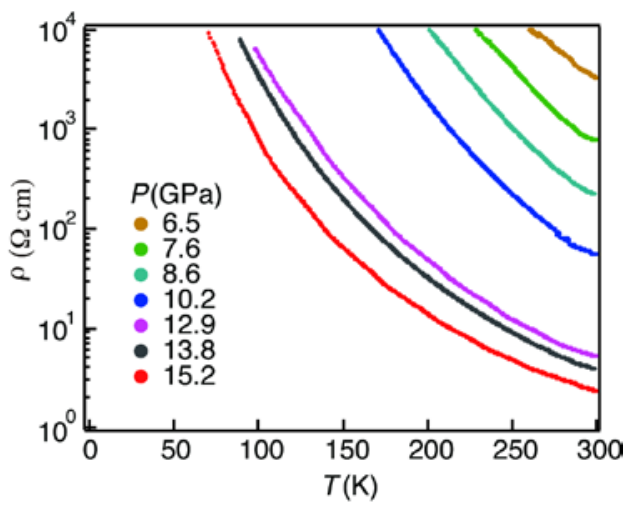

Fig. 22. Temperature dependence of the resistivity of radical dyad $\mathbf{3}$ along the $b$-axis at different pressures. Adapted with permission from ref. 64. Copyright (2016) American Chemical Society.

In order to understand the origin of the conducting behaviour in such neutral TTF-PTM dyads we performed theoretical calculations of the electronic structure and charge transfer based on predicted crystal structures of $\mathbf{3}$ at high pressure. The spin-polarized band structure calculations showed a gap close to the Fermi level $\left(\mathrm{E}_{\mathrm{F}}\right)$ at ambient pressure between the occupied bands with dominant TTF character (black lines in density of states) and the empty bands with dominant PTM character (Fig. 23). Blue and red colour indicates the different spin orientation. The gap between the bands diminished and closed at pressures higher than $6 \mathrm{GPa}$ observing that increasing the pressure the electronic bandwidth $W$ increased around one order of magnitude (from $0.1 \mathrm{eV}$ at $\mathrm{P}=0 \mathrm{GPa}$ to $0.6 \mathrm{eV}$ at $\mathrm{P}=8.6 \mathrm{GPa}$ ). 

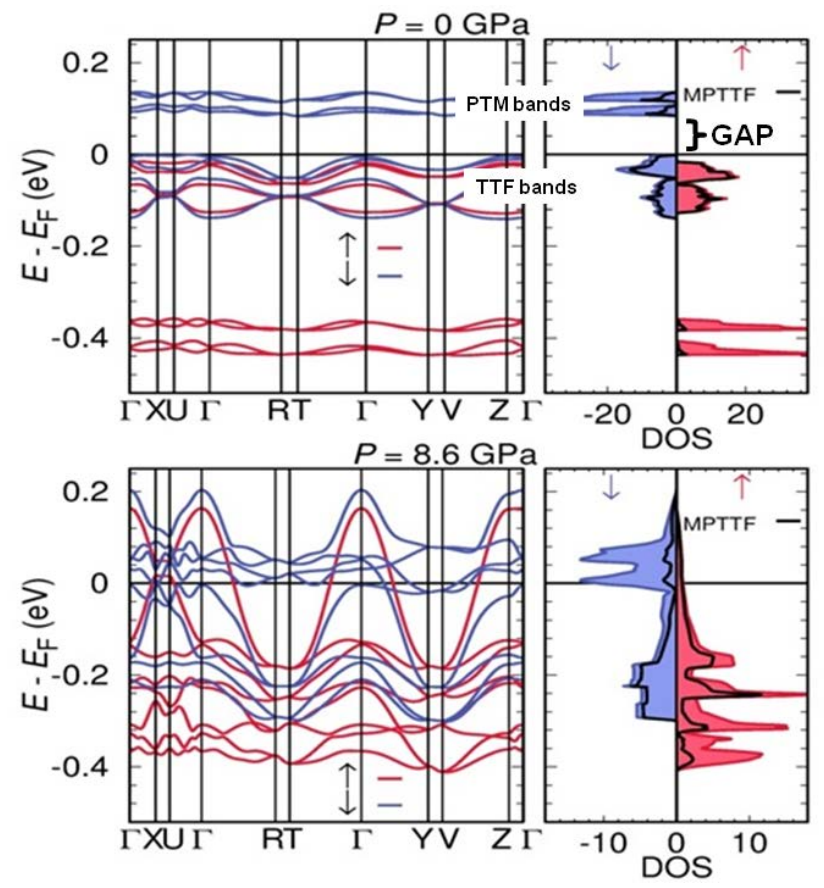

Fig. 23. Spin polarized electronic band structures and density of states (DOS) of radical dyad 3 at 0 GPa and 8.6 GPa. Adapted with permission from ref. 64. Copyright (2016) American Chemical Society.

On the other hand, the pressure evolution of charge transfer was calculated for the different units of the molecule distinguishing the MPTTF moiety as the electron donor and the PTM together with the bridge as the acceptor (Fig. 24). Increasing the pressure we observed that the charge transfer between the two units increases from $0.09 e$ - at $0 \mathrm{GPa}$ to $0.32 e$ - at $18 \mathrm{GPa}$ which is indicative of a partial selfdoping ability of the system. This charge reorganization occurring at high pressure could be related with a decreasing of couloumbic repulsion energy $U$ of the system when increasing the pressure. Moreover, high-pressure Raman spectroscopy also supported this charge reorganization in a similar pressure regime that was in fully agreement with the simulated Raman spectra from the predicted crystal structures at high pressure.

(a)
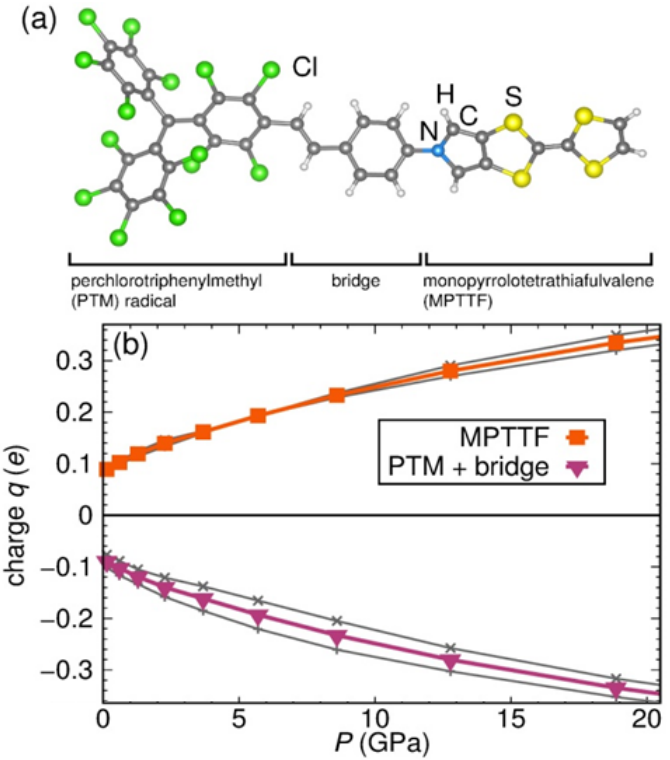

Fig. 24. Calculated evolution of excess charge within the units of radical dyad $\mathbf{3}$ with hydrostatic pressure. (a) The different selected parts of the radical dyad 3. (b) Excess of charge on the different units under high pressure. The grey lines denote the excess charge corresponding to the two non-equivalent molecules of $\mathbf{3}$ under pressure. Adapted with permission from ref. 64. Copyright (2016) American Chemical Society. 
Thus, we have reported the first example of a single-component conductor based on a TTF- $\pi$-PTM dyad and using a non-planar a spinlocalized radical. The origin of the conductivity was mainly attributed to the enhanced intermolecular interactions between the molecules due to the incorporation of the TTF moiety and to the enhanced charge delocalization of the system which is acting as internal partial doping. These results have been demonstrated to be a new proof of concept in order to design new neutral radical conductors and it may be possible to engineer new radical dyads which could be conducting without the need for applied pressure.

\section{Conclusions and outlook}

In summary, we have reported different examples of organic $D-\pi-A$ dyads based on the electron-donor TTF linked to the electron-acceptor PTM radical, as building blocks, through different conjugated bridges. These TTF- $\pi$-PTM dyads showed very interesting physical properties in solution and have been proposed as molecular switches. The interplay between the intra- and intermolecular CT processes played an important role for the self-assembly of such dyads and the understanding of such phenomenon is of high interest in order to design molecular conductors in solid state.

In the first example, TTF-PTM radical dyad 1 showed an intramolecular electron transfer process when tuning the polarity of the solvent. Indeed, the highly polar solvent DMF stabilized the formation of zwitterionic species at the same time that induced the self-assembly of the molecules forming diamagnetic dimers of zwitterions. Moreover, using the temperature as external stimulus was possible to switch the same system between diamagnetic dimers at room temperature and paramagnetic monomers at high temperature that exhibited different optical and magnetic properties.

On the other hand, we have extended the study of self-assembled properties of TTF- $\pi$-PTM to the solid state in order to develop new single-component molecular conductors. To this end, we investigated the supramolecular arrangement of different compounds paying a lot of attention to MPTTF-PTM radical dyad $\mathbf{3}$ that showed a crystal packing with segregated donor and acceptor units with S...S interactions between the TTF moieties. In order to promote conductivity on this system we applied high pressure observing semiconducting behaviour from $6.5 \mathrm{GPa}$. The origin of such conductivity was mainly attributed to the enhanced electronic bandwidth $W$ due to the short interaction between the molecules and to the charge reorganization occurring in the system decreasing the Coulombic repulsion $U$.

These studies demonstrated that organic TTF- $\pi$-PTM systems are very interesting materials in the field of molecular electronics finding application as molecular switches in solution or conductors in solid state. Tuning the bridge nature and the interplay between the intra- and intermolecular charge transfer offer excellent opportunities for developing new multifunctional molecular materials. Exploring new TTF- $\pi$-PTM derivatives could open the way to exploit and combine both the optical and magnetic properties in a cooperative manner for applications in spintronics and molecular electronics.

\section{Acknowledgements}

This work was supported by the EU Training Networks (ITN) "iSwitch" (GA No. 642196) and "NANO2FUN" (GA No. 607721) DGI (Spain) grant (BeWell; CTQ2013-40480-R), the Networking Research Center on Bioengineering, Biomaterials, and Nanomedicine (CIBER-BBN), and the Generalitat de Catalunya (grant 2014- SGR-17). ICMAB acknowledges support from the Spanish Ministry of Economy and Competitiveness, through the "Severo Ochoa" Programme for Centres of Excellence in R\&D (SEV- 2015-0496). M. S. is grateful to Spanish Ministerio de Educación, Cultura y Deporte for a FPU grant and he is enrolled in the Material Science Ph.D. program of UAB.

\section{Notes and references}

E. Coronado, A. Nuez and F. M. Romero, Solid State Sci., 2003, 5, 917-924.

L. Ouahab, Multifunctional Molecular Materials, CRC Press, 2013. 1954-1957.

Y. Wang, X. Tan, Y.-M. Zhang, S. Zhu, I. Zhang, B. Yu, K. Wang, B. Yang, M. Li, B. Zou and S. X.-A. Zhang, J. Am. Chem. Soc., 2015, 137, 931-939. 
W. R. Feringa, L.; Browne, Molecular Switches, Wiley, 2011.

J. L. Zhang, J. Q. Zhong, J. D. Lin, W. P. Hu, K. Wu, G. Q. Xu, A. T. S. Wee and W. Chen, Chem. Soc. Rev., 2015, 44, $2998-3022$.

A. Bousseksou, G. Molnár, L. Salmon and W. Nicolazzi, Chem. Soc. Rev., 2011, 40, 3313-3335.

O. Sato, J. Tao and Y.-Z. Z. Zhang, Angew. Chemie - Int. Ed., 2007, 46, 2152-2187.

C. W. Chu, J. Ouyang, J. H. Tseng and Y. Yang, Adv. Mater., 2005, 17, 1440-1443.

D. Small, V. Zaitsev, Y. Jung, S. V. Rosokha, M. Head-Gordon and J. K. Kochi, J. Am. Chem. Soc., 2004, 126, 13850-13858.

Y. Morita, S. Suzuki, K. Fukui, S. Nakazawa, H. Kitagawa, H. Kishida, H. Okamoto, A. Naito, A. Sekine, Y. Ohashi, M. Shiro, K. Sasaki, D. Shiomi, K. Sato, T. Takui and K. Nakasuji, Nat. Mater., 2008, 7, 48-51.

Y. Morita, S. Suzuki, K. Sato and T. Takui, Nat. Chem., 2011, 3, 197-204.

R. G. Hicks, Nat. Chem., 2011, 3, 189-191.

M. E. Itkis, X. Chi, A. W. Cordes and R. C. Haddon, Science, 2002, 296, 1443-1445.

S. K. Pal, P. Bag, A. Sarkar, X. Chi, M. E. Itkis, F. S. Tham, B. Donnadieu and R. C. Haddon, J. Am. Chem. Soc., 2010, 132, 1725817264.

J. M. Rawson and F. Palacio, Structure, 2001, 100, 94-108.

J. L. Brusso, O. P. Clements, R. C. Haddon, M. E. Itkis, A. A. Leitch, R. T. Oakley, R. W. Reed and J. F. Richardson, $2004,8256-8265$.

R. C. Haddon, Nature, 1975, 256, 394-396.

S. K. Pal, M. E. Itkis, F. S. Tham, R. W. Reed, R. T. Oakley and R. C. Haddon, Science, 2005, 309, $281-284$.

S. K. Mandal, S. Samanta, M. E. Itkis, D. W. Jensen, R. W. Reed, R. T. Oakley, F. S. Tham, B. Donnadieu and R. C. Haddon, J. Am. Chem. Soc., 2006, 128, 1982-1994.

S. K. Pal, M. E. Itkis, F. S. Tham, R. W. Reed, R. T. Oakley and R. C. Haddon, J. Am. Chem. Soc., 2008, 130, 3942-3951.

A. Mailman, S. M. Winter, X. Yu, C. M. Robertson, W. Yong, J. S. Tse, R. a. Secco, Z. Liu, P. a. Dube, J. a K. Howard and R. T. Oakley, J. Am. Chem. Soc., 2012, 134, 9886-9889.

X. Yu, A. Mailman, K. Lekin, A. Assoud, C. M. Robertson, B. C. Noll, C. F. Campana, J. a K. Howard, P. a. Dube and R. T. Oakley, J. Am. Chem. Soc., 2012, 134, 2264-2275.

J. W. L. Wong, A. Mailman, K. Lekin, S. M. Winter, W. Yong, J. Zhao, S. V. Garimella, J. S. Tse, R. a. Secco, S. Desgreniers, Y. Ohishi, F. Borondics and R. T. Oakley, J. Am. Chem. Soc., 2014, 136, 1070-1081.

L. Yao, S. Zhang, R. Wang, W. Li, F. Shen and B. Yang, Angew. Chemie - Int. Ed., 2014, 53, $2119-2123$.

F. Bures, RSC Adv., 2014, 4, 58826-58851.

R. M. Metzger, Chem. Rev., 2003, 103, 3803-3834.

A. A. Gorshelev, A. V Naumov, Y. Eremchev, Y. G. Vainer, L. Kador and J. Kohler, Chem. Phys. Chem., 2010, 11, $182-187$.

I. Ratera and J. Veciana, Chem. Soc. Rev., 2012, 41, 303. 
Y. Yamashita and M. Tomura, J. Mater. Chem., 1998, 8, 1933-1944.

J. J. Bergkamp, S. Decurtins and S.-X. Liu, Chem. Soc. Rev., 2015, 44, 863-874.

Y. Geng, R. Pfattner, A. Campos, J. Hauser, V. Laukhin, J. Puigdollers, J. Veciana, M. Mas-Torrent, C. Rovira, S. Decurtins and S.-X. Liu, Chem. Eur. J., 2014, 20, 7136-7143.

J. L. Segura and N. Martn, Angew. Chemie - Int. Ed., 2001, 40, 1372-1409.

N. Martín, Chem. Commun., 2013, 49, 7025-7.

P. Batail, Chem. Rev., 2004, 104, 4887-4890.

F. Wudl, G. M. Smith and E. J. Hufnagel, J. Chem. Soc., Chem. Commun., 1970, 1453-1454.

D. Canevet, M. Sallé, G. Zhang, D. Zhang and D. Zhu, Chem. Commun., 2009, 7345, 2245-2269.

F. Pop and N. Avarvari, Chem. Commun., 2016, 52, 7906-7927.

G. Ho, J. R. Heath, M. Kondratenko, D. F. Perepichka, K. Arseneault, M. Pézolet and M. R. Bryce, Chem. - A Eur. J., 2005, 11, 29142922.

S. V. Rosokha and J. K. Kochi, J. Am. Chem. Soc., 2007, 129, 828-838.

P. T. Chiang, N. C. Chen, C. C. Lai and S. H. Chiu, Chem. - A Eur. J., 2008, 14, 6546-6552.

M. Yoshizawa, K. Kumazawa and M. Fujita, J. Am. Chem. Soc., 2005, 127, 13456-13457.

J. Veciana and I. Ratera, Radicals : Towards Multifunctional Molecular Materials, Wiley, 2010.

I. Ratera, D. Ruiz-Molina, F. Renz, J. Ensling, K. Wurst, C. Rovira, P. Gütlich and J. Veciana, J. Am. Chem. Soc., 2003, 125, $1462-1463$.

I. Ratera, C. Sporer, D. Ruiz-Molina, N. Ventosa, J. Baggerman, A. M. Brouwer, C. Rovira and J. Veciana, J. Am. Chem. Soc., 2007, 129, 6117-6129.

G. D’Avino, L. Grisanti, J. Guasch, I. Ratera, J. Veciana and A. Painelli, J. Am. Chem. Soc., 2008, 130, 12064-12072.

L. Grisanti, G. D’Avino, A. Painelli, J. Guasch, I. Ratera and J. Veciana, J. Phys. Chem. B, 2009, 113, 4718-4725.

J. Guasch, L. Grisanti, S. Jung, D. Morales, G. D’Avino, M. Souto, X. Fontrodona, A. Painelli, F. Renz, I. Ratera and J. Veciana, Chem. Mater., 2013, 25, 808-814.

M. Souto, D. C. Morales, J. Guasch, I. Ratera, C. Rovira, A. Painelli and J. Veciana, J. Phys. Org. Chem., 2014, 27, 465-469.

G. D’Avino, L. Grisanti, A. Painelli, J. Guasch, I. Ratera and J. Veciana, CrystEngComm, 2009, 11, 2040-2047.

S. Nishida, Y. Morita, K. Fukui, K. Sato, D. Shiomi, T. Takui and K. Nakasuji, Angew. Chemie Int. Ed., 2005, 44, 7277-7280.

J. Guasch, L. Grisanti, V. Lloveras, J. Vidal-Gancedo, M. Souto, D. C. Morales, M. Vilaseca, C. Sissa, A. Painelli, I. Ratera, C. Rovira and J. Veciana, Angew. Chemie - Int. Ed., 2012, 51, 11024-11028.

J. Guasch, L. Grisanti, M. Souto, V. Lloveras, J. Vidal-Gancedo, I. Ratera, A. Painelli, C. Rovira and J. Veciana, J. Am. Chem. Soc., 2013, 135, 6958-6967. 
M. Souto, J. Guasch, V. Lloveras, P. Mayorga, J. T. López Navarrete, J. Casado, I. Ratera, C. Rovira, A. Painelli and J. Veciana, J. Phys. Chem. Lett., 2013, 4, 2721-2726.

O. Elsner, D. Ruiz-Molina, J. Vidal-Gancedo, C. Rovira and J. Veciana, Chem. Commun., 1999, 579-580.

F. Otón, V. Lloveras, M. Mas-Torrent, J. Vidal-Gancedo, J. Veciana and C. Rovira, Angew. Chemie - Int. Ed., 2011, 50, 10902-10906.

J. Calbo, J. Aragó, F. Otón, V. Lloveras, M. Mas-Torrent, J. Vidal-Gancedo, J. Veciana, C. Rovira and E. Ortí, Chem. - A Eur. J., 2013, 19, 16656-16664.

T. Sugawara and M. M. Matsushita, J. Mater. Chem., 2009, 19, 1738. Veciana, Chem. - A Eur. J., 2015, 21, 8816-8825. 